\title{
Health Informatics System for Screening Arboviral Infections in Adults
}

\author{
Chinecherem Umezuruike \\ Kampala International University, Kampala, Uganda \\ E-mail: umezuruikechinecherem@gmail.com \\ Dr. Wilson Nwankwo \\ Edo University Iyamho, Edo State, Nigeria \\ E-mail: Nwankwo.wilson@edouniversity.edu.ng \\ Prof. Samuel O. Okolie \\ Babcock University Ilishan, Ogun State Nigeria \\ E-mail: samuelokolie2003@yahoo.com \\ Dr. Adewale O. Adebayo \\ Babcock University Ilishan, Ogun State Nigeria \\ E-mail: adebayoa@babcock.edu.ng \\ Dr. Joshua V. Jonah \\ Babcock University Ilishan, Ogun State Nigeria \\ E-mail: jvjoshua@yahoo.co.uk \\ Habiba Ngugi \\ Kampala International University, Kampala, Uganda \\ E-mail: habiba.ngugi9@gmail.com
}

Received: 18 December 2018; Accepted: 20 January 2019; Published: 08 March 2019

\begin{abstract}
Health Informatics (HI) has played vital roles in the management of several diseases especially in the tropics. It has revolutionized the mainstream healthcare and healthcare delivery system. This paper applies the principle of Health Informatics to addressing the detection and management of arboviruses particularly Zika and Dengue viruses around which the aetiology of Zika Virus Disease and Dengue fever revolves. In this paper, the object-oriented approach was employed to study the fundamental procedures in the detection and management of arboviral infections. The study culminated into modelling of knowledge-based prototype system for screening patients in incidence areas. Existing knowledge on the management of arbovirus infections was complemented with purposive sampling of two specialist infectious diseases facilities in Nigeria. The health informatics prototype is christened NCliniSoft Diagnostic ZikaSol and is composed of five components validated through expert-driven differential diagnostic procedures. The prototype was evaluated to test for usability, diagnostic consistency, user acceptance and satisfaction. The prototype performs a differential screening between Dengue fever and Zika Virus disease using the Bayesian probabilities complemented by situational constructs. The result of each screening
\end{abstract}

process is an automated diagnostic report that shows the status of the patient. Computed result showed high level of efficiency and acceptability.

Index Terms-Arboviruses, Clinical Differential Diagnostic System, Dengue fever, Health Informatics, Zika Virus.

\section{INTRODUCTION}

Health Informatics is an interdisciplinary study of the design, development, adoption, and application of ITbased innovations in healthcare services delivery, management, and planning [1]. HI has played key roles in the eradication of small pox in the 1970s and management of Severe Acute Respiratory Syndrome (SARS) in 2003 [2]. This paper applies the basic principles of Health informatics to addressing the detection and management of arboviruses especially in the differential screening of Flaviviral and Alphaviral infections.

In this paper, a differential diagnostic system was developed, by using goal driven, backward chain inference approach and integrating a bayesian probabilistic rule that determines prevalence of specific 
disease in the human system of a suspected infected patient.

Rest of the paper is organized as: Section II presents related work. Section III disclosed the materials and methods deployed to get results. Section IV is dedicated to the results. Section V discussed the operations of the developed system.

\section{A. Statement of the Problem}

Epidemiological studies had revealed that environmental and human behavioural risk factors in areas with recent Zika outbreaks like Brazil are similar to those found in Nigeria and other tropical environment hence the likelihood of the outbreak of the disease anytime. In addition, inadequate diagnostic testing and the absence of systematic surveillance are also limitations which make diagnosis difficult hence the urgent need for adequate preventive

\section{B. Aim and Objectives of the Study}

The aim of this paper is to present functional and useful software that can be put into use at any time, in any health facility, for managing arboviral infections. The objectives of this paper are to:

1. Present detailed design specifications for an arboviral detection software system

2. Express the design specifications in terms of algorithms for implementation.

3. Demonstrate the implementation procedures as well as the results of a functional medical diagnostic detection system.

\section{Significance of the Study}

This paper has the following significance:

1. It provides an authentic disease detection solution that could be used by primary health care facilities to quickly screen patients for Zika and Dengue viral infections.

2. Like every other software engineering process, detailed specifications of the design and implementation procedures enables reproducibility of the system as well as aid the maintenance and extension of the system.

3. Provide a basis for future studies on the incorporation of advanced software intelligence into clinical diagnostic procedures especially for infectious diseases that are very likely to spur an epidemic

\section{RELATED WORKS}

Flaviviruses refer to a subcategory of arboviruses also known as arthropod-borne viruses [3, 4] and transmitted to man by insects such as mosquitoes, ticks, sandflies or biting midges. The traditional way of transmission to humans is often through bites. Though insect bites are the commonest way of transmission to humans, these viruses can also spread through: blood transfusion, sexual act, organ transplantation, pregnancy, childbirth (from mother to child), etc. Members of the Flavivirus family are: Zika Virus, Yellow fever virus, West Nile virus, Dengue fever virus, Japanese encephalitis virus, etc. Among these viruses, Zika, and Yellow fever are more pronounced. Another category of arboviruses worth discussing is the alphaviruses. Some members of alphaviruses such as Dengue virus do share similar signs and symptoms with the flaviviruses.

Zika Virus Disease (ZVD) was first identified in 1947 in the Zika forest of Ugandan in East Africa. Its aetiologic agent is specie of mosquito known as aedes aegypti. In 1952 and 1954 it was diagnosed to have infected humans in Uganda, Tanzania and Nigeria [5,6]. In 2007, there was an outbreak of Zika Virus infection in Yap Federal State of Micronesia [7,8] and Brazil with 30,000 reported cases of Zika Virus Disease in 2015 $[9,10]$. With the outbreak of ZVD in 2015 in Brazil, health implication awareness was created by World Health Organisation [11] and several Zika Virus models were developed to study biological facts of the Virus reproductive processes, transmission and favourable prevention process $[12,13,14,15,16]$.

Based on prevalence and need, medical diagnostic systems have been developed for common diseases [17], for viral infections[18] excluding Zika, diagnosis of depression [19]. Abu, Al-dahdooh, Mustapher and ElNaffer [20] had proposed Expert system for medical diagnostic assistance (ESMDA) covering six diseases: Cold and Flu, Cough, fever, Ear and Eye problems. Automated Diagnostic systems have always been developed to cater for specific diseases. However, most automated diagnostic or detection systems are not always designed to recognize new outbreaks of diseases.

With the likelihood of outbreak of Zika Virus and its diagnostic difficulties due to symptomatic similarities with other arboviruses like Dengue, Chikungunya, West Nile disease, and mode of transmission [4], it has become imperative to develop a screening or precursor clinical differential diagnostic system for the notable Flaviviral infections.

\section{MAterials AND Methods}

\section{A. Materials}

The materials used are:

1. Survey questionnaires

2. PC with 25.GHZ quad-core processor, 16GB RAM, and 500 TB Hard disk, and running Microsoft Windows 10 Operating System.

3. Microsoft Visual Studio 2015

4. Microsoft SQL Server 2014 Enterprise

5. Erwin Data modeller

6. Data collection Notepad

7. Techno Tablet running Android 7.0

\section{B. Data Collection and System Analysis}

The method employed in this study is based on the 
tenets of the Object-oriented Analysis and Design Methodology (OOADM). Figure 1 shows the study procedure. First, a detailed investigation is conducted on the prevalence and incidence of arboviruses having regarded the perceived likelihood of their occurrence and the danger such infections pose in the tropics. A desk search was conducted over the Internet and in local public libraries. In carrying out the desk search, 200 hundred related works were searched out from the internet and online databases using Google, Bing, ask.com, who.int, cdc.gov, ncdc.gov.ng and PubMed with such keywords like Arbovirus diseases, Dengue fever, ZVD, Health informatics, Health information system, Clinical decision support system and Clinical differential diagnostic system. Out of the 200 materials retrieved 50 were found to be very relevant to the study. The Information provided through literature was complemented with purposeful sampling of two secondary public healthcare facilities in Nigeria. The selection of public health facilities was based on the WHO risk framework for ZVD [21].

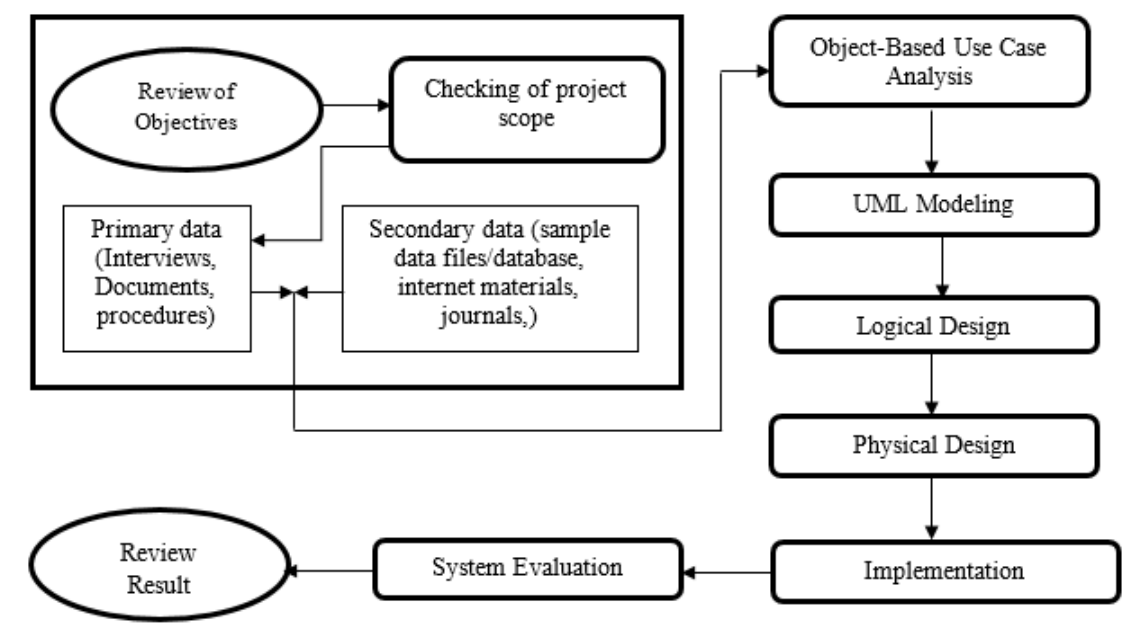

Fig.1. Research Procedure

The Medical Directors of the said facilities were duly informed through a letter of request to use their facilities as resource centre for the study. The requests were granted accordingly. Following the request was the design of experiments (questionnaires, analytic diagrams, models, and simulation) that would assist in realizing the intended solution. Domain Knowledge data were gathered through discussion with four Infectious disease specialists as well as from the World Health Organization

The collected data provided the inputs for an objectoriented use-case analysis prior to the design of the proposed system. As Kaasbøl [22] puts it, "the hard core of object-orientation consists of the following: encapsulated objects with properties and behaviour, classes of objects, and inheritance of general properties and behaviour to specialized classes". The substance of the process is organized as system components, called objects. A measurable property of the substance is a property of an object. Transformation of state is regarded as actions by objects. Mathiasen, et al. [23] had laid emphasis on the importance of objects, states, and behavior, as the most suitable approach for describing most phenomena that can be expressed in natural language. The main difference between object-oriented methodology and others lie in the fact that while using the object-oriented approach, requirements are structured around objects in such a way that both behaviors (processes) and states (data) are integrated and modeled after real world objects that lie with the problem domain and by extension the system environment, traditional approaches treat processes and data as two separate entities.

\section{Programming paradigm}

The Model-View-Controller (MVC) approach was adopted in the program implementation process. The MVC model separates an application program into three distinct layers, each with a specific role.

1. Model (Data Access Tier/Data access) layer: an implementation of a layer of code that provides access to the database. It is important to keep the data access code in a separate layer in order to support multiple database management systems, automated data auditing, connection management, hiding of connection strings, and retrieval of identity values.

2. Controller Logic layer: Also called the user interface (UI) process logic or presentation logic layer. In a web-based application such as the one proposed in this paper, the controller logic handles the flow of the web pages as a user performs various operations.

3. View (Web presentation Layer): This layer consists of the various interfaces and forms that the user of the system can easily interact with. The view layer contains the code that interacts with the controller objects.

\section{Business Logic}

There are two flaviviral infections that present overlapping signs and symptoms namely: Zika, and 
Degue. The third arboviral infection is Chikugunya viral infection, however, Chikugunya belongs to the alphavirus subclass of arboviruses unlike Zika and Dengue. Zika and Dengue share the closest set of signs and symptoms. Nevertheless, it does not follow that there isn't an overlap between the symptoms and signs accompanying these two infections. To effect a differential detection, the set of all possible symptoms of these two diseases are compiled following the WHO specifications. The business logic for differentiation between the two is built using the Bayes' rule.

\section{Bayes' rule}

Rationale 1: Let $\mathrm{V}_{1}, \mathrm{~V}_{2}, \mathrm{~V} 3, \ldots \mathrm{V}_{\mathrm{n}}$ be the set of $\mathrm{n}$ mutually exclusive flaviviral diseases. Let $\mathbf{S}$ be a set of signs and symptoms accompanying any of $\mathbf{V}_{\mathrm{i}}$. Baye's rule is satisfied by (1).

$$
\begin{aligned}
& \mathbf{P}\left(\mathbf{V}_{\mathrm{i}} \mid \mathbf{S}\right)=\quad \mathrm{P}\left(\mathrm{S} \mid \mathrm{V}_{\mathrm{i}}\right) \mathrm{P}\left(\mathrm{V}_{\mathrm{i}}\right) \\
& \sum_{j=i}^{n} \mathrm{P}(\mathrm{Vj}) \mathrm{P}(\mathrm{S} \mid \mathrm{Vj})
\end{aligned}
$$

Where $i=1,2,3 \ldots n$

$$
\text { Alternatively } \mathbf{P}\left(\mathbf{V}_{\mathrm{i}} \mid \mathbf{S}\right)=\mathrm{P}\left(\mathrm{S} \mid \mathrm{V}_{\mathrm{i}}\right) \mathrm{P}\left(\mathrm{V}_{\mathrm{i}}\right) / \mathrm{P}(\mathrm{S})
$$

Therefore, $\mathrm{P}\left(\mathrm{V}_{\mathrm{i}} \mid \mathrm{S}\right)$ implies the probability of $\mathrm{V}_{\mathrm{i}}$ th flaviviral infection given the set of signs and symptoms $\mathbf{S}$; or in other words, the $\mathbf{P}\left(\mathbf{V}_{\mathrm{i}} \mid \mathbf{S}\right)$ is the posterior probability of $\mathbf{V}_{\mathbf{i}}$ given $\mathbf{S}$ because it does depend on having information about $S$. $\mathbf{P}\left(\mathbf{V}_{\mathbf{i}}\right)$ is the marginal or prior probability of $\mathbf{V}_{\mathbf{i}}$, since it is the probability of $\mathbf{V}_{\mathbf{i}}$ prior to having any information about $\mathbf{S} . \mathbf{P}(\mathbf{S})$ is the marginal or prior probability of $\mathrm{S}$. The term $\mathrm{P}\left(\mathrm{S} \mid \mathrm{V}_{\mathrm{i}}\right)$ is the likelihood function for $\mathrm{S}$ given $\mathrm{V}_{\mathrm{i}}$.

\section{Rationale 2:}

Let $\mathrm{A} \Rightarrow$ Patient

Let $\mathrm{B}=>$ Symptom/Sign of a Flaviviral infection such as ZVD, Dengue, etc.

Let the probability that a patient is infected $=P(A)$

Let the probability that the patient exhibited the symptoms $=P(B)$

Let the probability that the patient is infected and there are identified symptoms $=P(A \cap B)$

Let the probability that a patient is diagnosed positive based on identified symptom $=P(A / B)$

$$
\begin{aligned}
& P(A)=P(A \cap \mathbf{B}) \\
& P(B)=P(B \cap \mathbf{A})
\end{aligned}
$$

Applying probability multiplication rule where

$$
P(A \cap B)=P(A) \cdot P(B / A)
$$

Substituting (5) in (3)

$$
P(A)=P(A) \cdot P(B / A)
$$

Which implies that

$$
P(A / B)=P(A) \cdot P(B / A)
$$

where

$$
P(A)=P(A / B)
$$

$$
\text { Assuming } P(A / B)=P(B / A)
$$

then

$$
P(B) \cdot P(A / B)=P(A) \cdot P(B / A)
$$

Hence

$$
P(A / B)=\frac{P(A) \cdot P(B / A)}{P(A)}
$$

Suppose $B$ occurs with an exclusively mutual condition $B_{1}$ provided $P(B) \geq 0$

$$
A=(A \cap B) \cup\left(A \cap B_{1}\right)
$$

Then

$$
P(A)=P(A \cap B) \cup P\left(A \cap B_{1}\right)
$$

Applying addition rule

$$
P(A)=P(A \cap B)+P\left(A \cap B_{1}\right)
$$

Substituting (10) in (8)

Hence

$$
P(A / B)=\frac{P(A) \cdot P(B / A)}{P(A \cap B)+P\left(A \cap B_{1}\right)}
$$

Then

$$
P(A / B)=\frac{P(A) \cdot P(B / A)}{\left(P(A) \cdot P(B / A)+P(A) \cdot P\left(B_{1} / A\right)\right)}
$$

\section{Symptomatic analysis}

Symptomatic analysis is the centre of the logic of the intelligence engine. This is because; an overlap of signs and symptoms exists across the flaviviral and alphaviral infections. That is also why there is a need for a historical reference to previously detected cases during the internal analysis of a new case. Results from the mining of 
historical data on cases of the infections are also considered and are used to enhance the probabilistic analysis. Other factors that are considered in the analysis are: geographical location of the person/patient, travels made within a given period of time to some places categorized as areas of high incidence, etc. The foregoing factors are weighted according to their sensitivity values. For instance, a location with likelihood of outbreaks attracts a higher weight and lower weight otherwise. In the case of travels to places with high likelihood of incidence within the last seven days, a maximum weight of 5 is attached whereas a trip to places of low likelihood of incidence within the last 7 days attracts a weight of 0 .

\section{Modeling the System}

The proposed diagnostic system model integrates five components. These components are the user interface components, the Database and DataMart, the extracttransform-load (ETL) component, the intelligent component and the.

1) The User interface component has 5 different modules or subsystems. These modules include the Patient management, Staff management, Help, and report.

2) The database component consists of the database management system (DBMS), the Database, and the DataMart. Information stored in the staging database is periodically transferred to the DataMart where it is made available to users of the facility for training and research.

3) The Extract Transform and Load (ETL) Component: extracts evidence based information from the database, transforms it to a specified format and loads them into a temporary storage within the system for further use. In the case of this system it extracts patients' symptoms and signs and compares it with the stored information in the inference engine to diagnose the disease in question and then loads the outcome to a temporary database.

4) Intelligence mining component: The information extracted from the database is transformed and loaded to the intelligence mining component. The information is analyzed giving a differential diagnosis of the prevalent disease based on signs and symptoms exhibited by the patient and captured into the database. The business logic makes use of the Bayesian probabilities. As a differential diagnostic model it compared the patients' symptoms and signs with the system knowledge base and makes prediction based on percentage ratios. The disease with the highest percentage is considered present in the patient and subject to a laboratory test for confirmation.

\section{RESULTS}

\section{A. The Diagnostic Model}

\section{Logical model}

The logical model of the system is shown in Figure II. It does show the attributes and relationships of the various concepts and the data characteristics of the various data elements that may be captured against the concepts. In other words, the logical model specification shows the various objects and the basic data type associated with every attribute of the given object. These data characteristics form the cornerstone of the final database model usually represented with the physical data model. The logical model is further extended to show the primary key or attribute(s) that uniquely identify each object. These primary keys are underlined in the logical diagrams in Figure II. For instance, registration number of a patient $(\operatorname{RegNo})$ is an attribute of a patient that uniquely identifies and distinguishes one patient from other patients.

\section{Interaction model}

The interaction among actors is expressed via the sequence diagram shown in Figure III. In the diagram, the actors and their activities are represented at behavioural level. Five actors are identified consisting of four human actors (nurse, patient, doctor, and account) and a non-human actor (the database). The activities carried out and their timelines are represented with arrowed lines.

\section{The Component Model}

The component model is shown in Fig.4. The component model comprises all the subsystems in the prototype diagnostic system. The component model includes: the Application frontend, Database, DataMart, Intelligent Miner, and the ETL module

\section{System Implementation}

The user interfaces are shown in [5-8. The system is web-based and accessible over the internet using all common web browsers. Fig 5. shows the user login page through which a user signs into the system. When the user signs in, the system authenticates the user based on the registered criteria and opens the appropriate page based on the user role.

Fig.6., shows the case history window which allows the clinician to fetch the patient's biodata and take case history. The case history window is made up of prelimanary test windows, signs and symptoms. It affords the clinician the opportunity to select applicable signs and symptoms as well as input those that are not presently on the system. Fig 7., shows the Diagnostic window. The diagnostic window displays the results of 
the anlalysis based on the algorithmic computations on the symptoms, signs, and other conditions detected during the case history taking process. Fig. 8. shows the opinion of the detection system in the form of a diagnostic report.

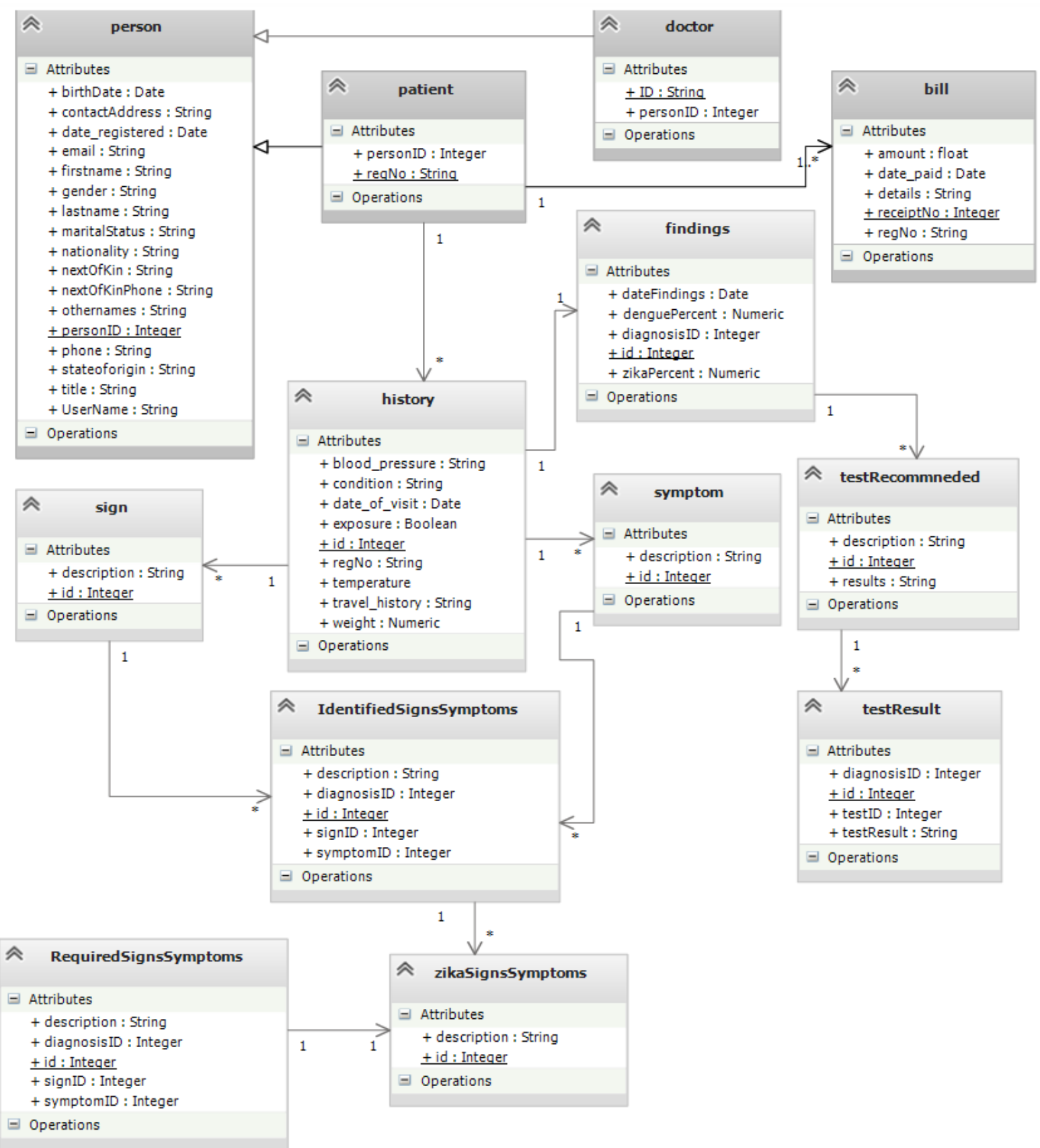

Fig.2. The Logical Model of the Diagnostic System 


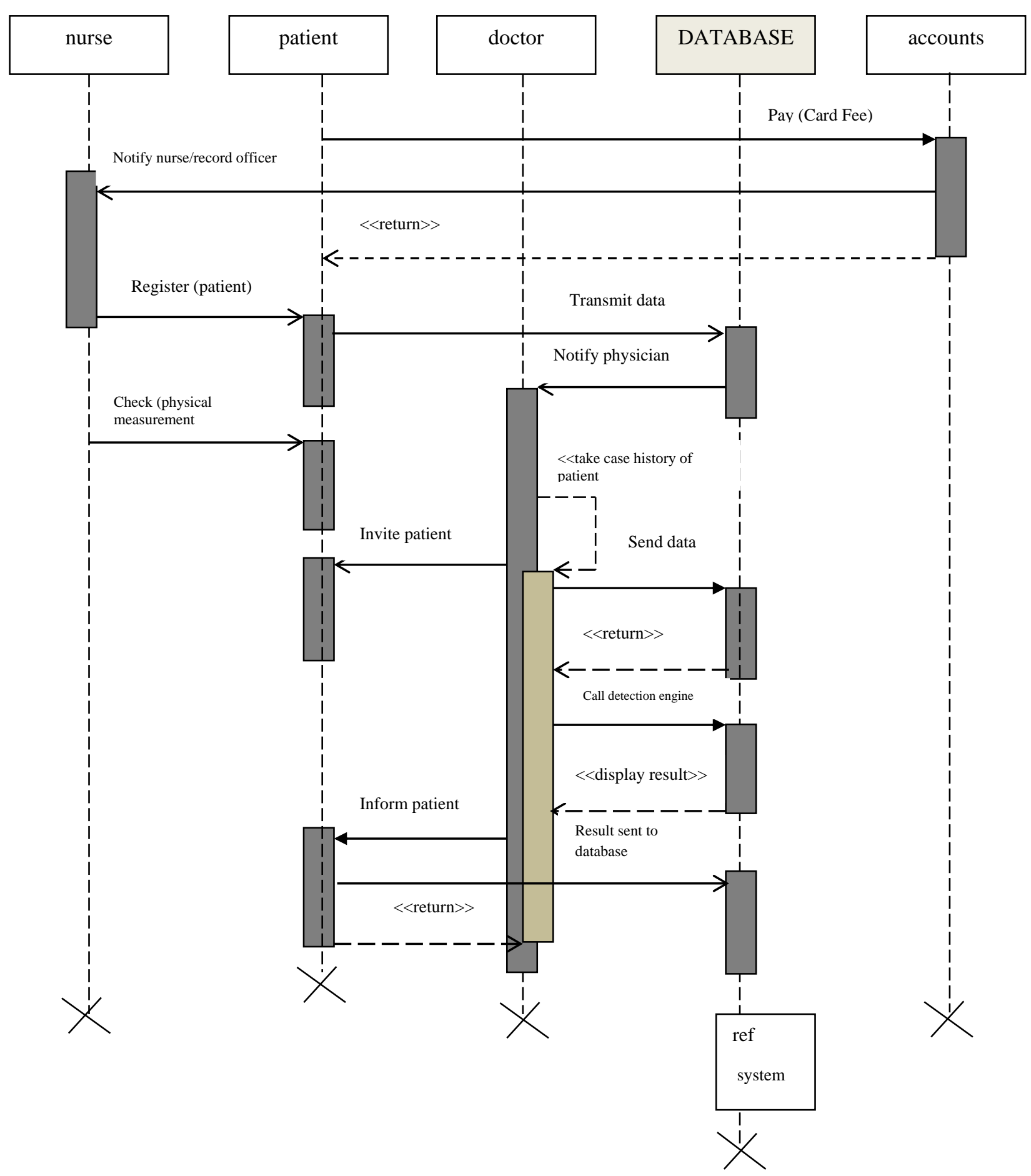

Fig.3. The System Sequence Diagram 


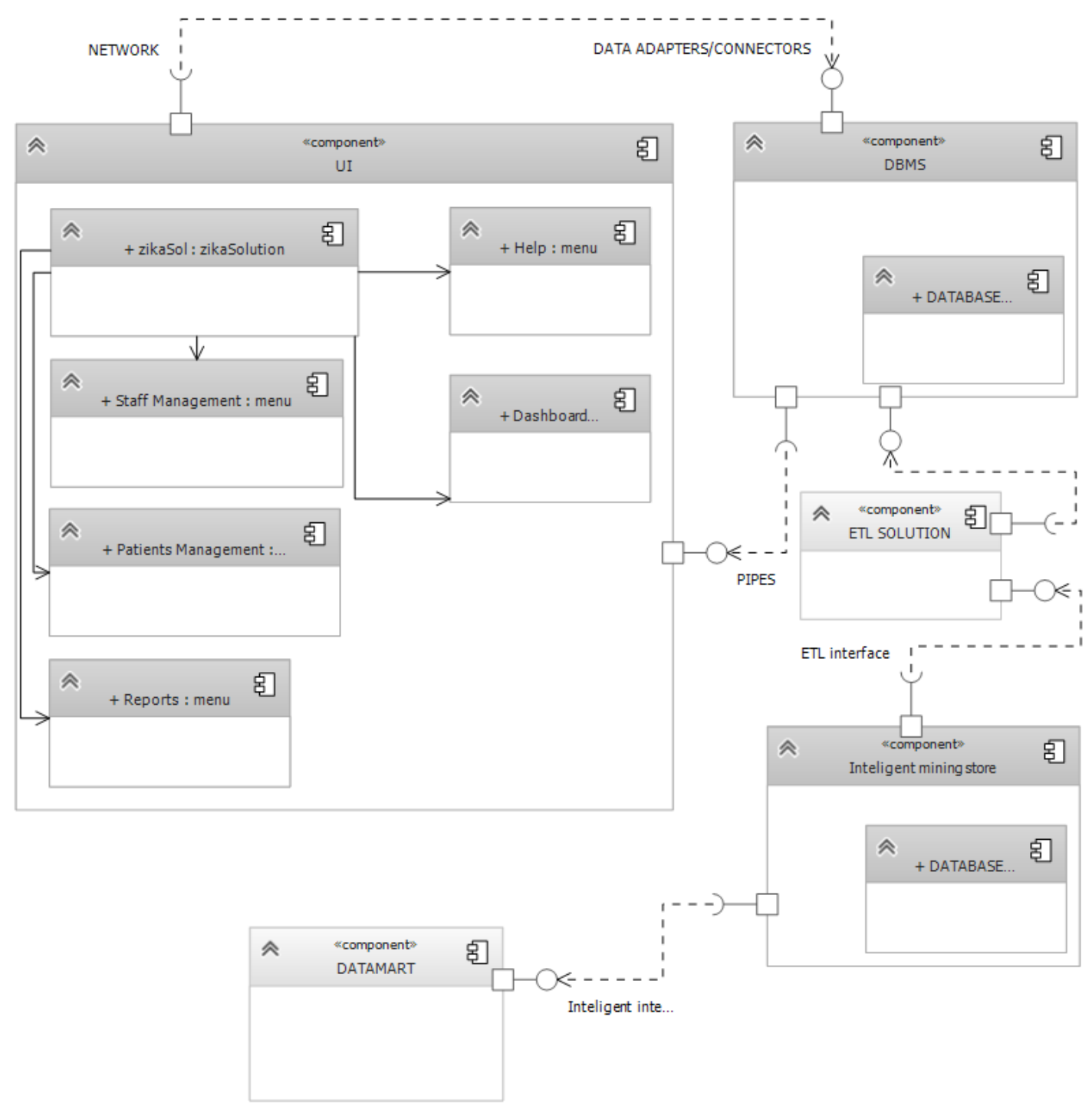

Fig.4. The Component Model of the System

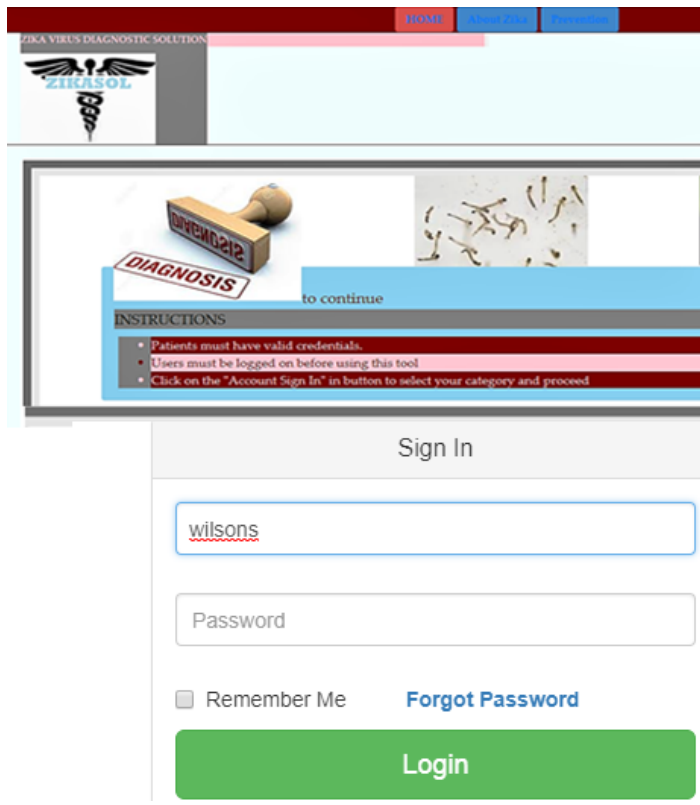

Fig.5. User Login Page 


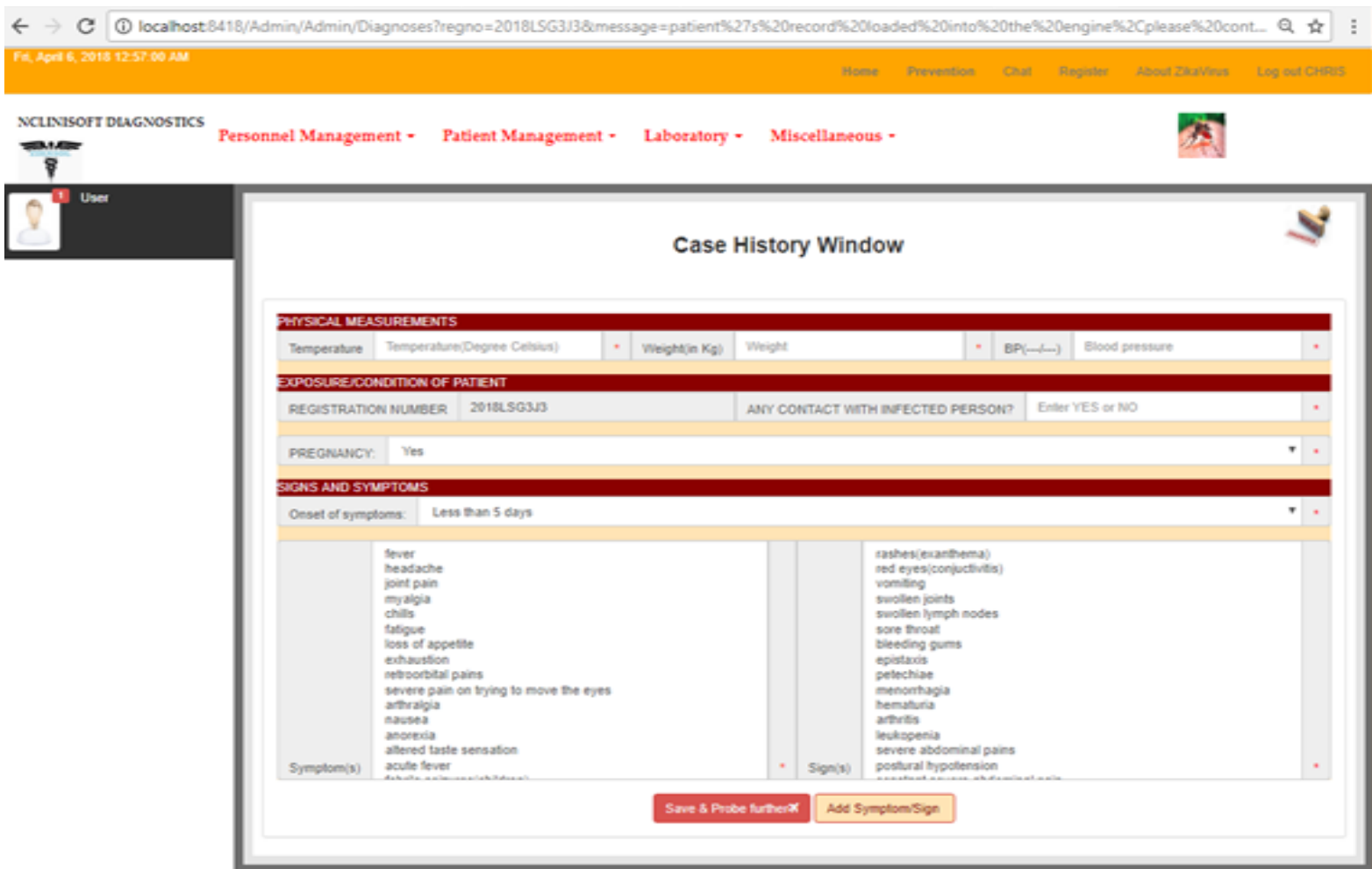

Fig.6. Case history Window

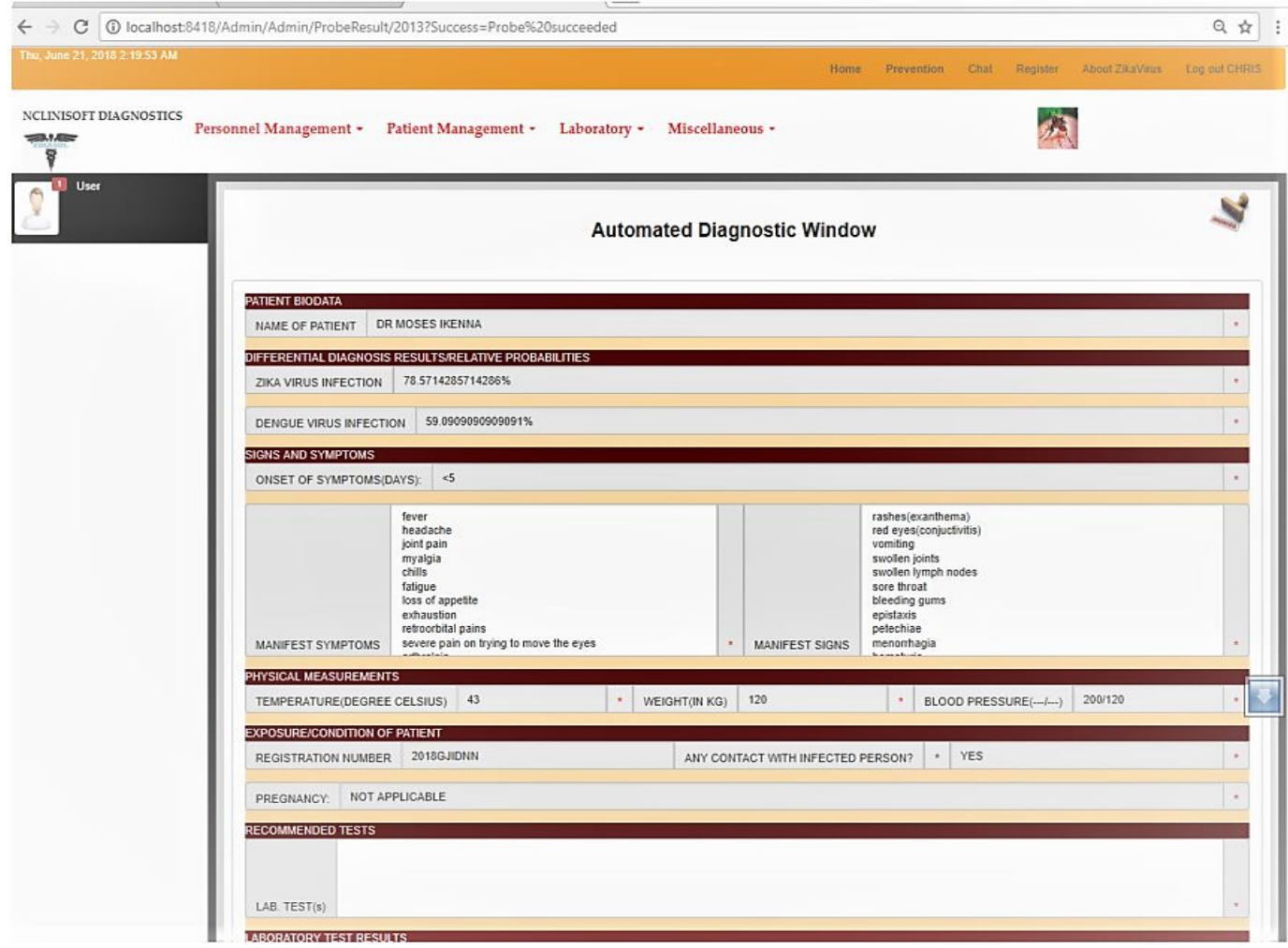

Fig.7. Diagnostic Window 


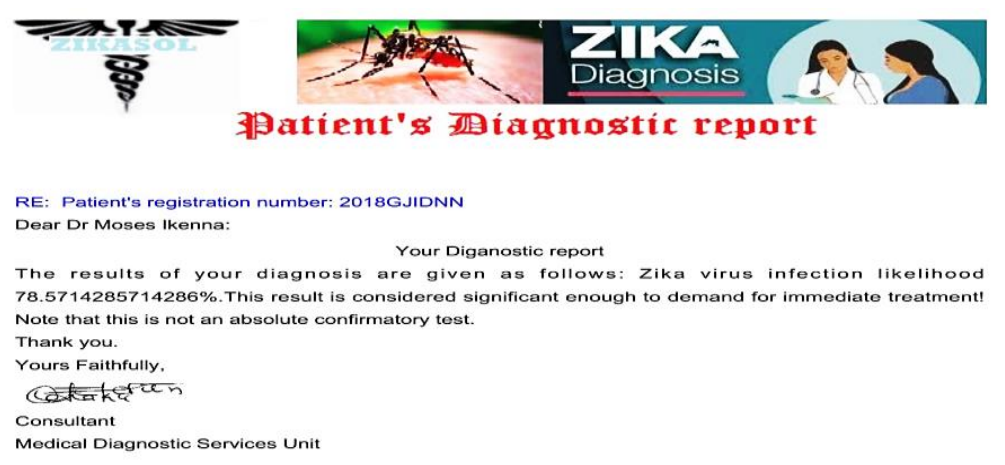

Medical Diagnostic Services Unit

Fig.8. Patient Diagnostic Report

\section{DISCUSSION}

\section{A. Testing and Evaluation}

The prototype system was tested for efficiency, user satisfaction and performance. Brokes system usability scale questionnaire was used to collect data from the users and the zika virus disease and dengue fever symptoms identified by WHO as gold reference standard, was benchmark for the differential diagnostic ability testing. Brokes questionnaire is a ten item Likert scale questionnaire used to assess usability of the prototype system. The responses from the respondents are tabulated in Table 1. All disagreed and agreed were merged together to give a value that indicates users perception of the system as relates to item tested and tabulated in Table II. For clarity and better understanding a multiple bar chart was used for comparative analysis of the items and shown as Fig.9. From Table 2, it was seen that the system passed the usability test based on the responses by the users.

\section{B. Performance Evaluation against the Gold Reference Standard}

The performance evaluation was done using database updating and differential diagnostic ability, and report generation as metrics. Gold reference standard was the basics for differential diagnosis. The gold reference standard is the factual information identified by the centre for disease control in 2016 as the signs and symptoms of Zika Virus disease and dengue fever. The system obtained the status of infection of a patient through a differential diagnosis by comparing the signs and symptoms that are already built into the database. The confirmation of the result was an automated diagnostic result that states in percentage level of infection of disease. The report of 10 patients is tabulated in Table 3., as confirmation of the differential diagnostic ability of the system.

Table 1. Responses on System Usability by User

\begin{tabular}{|c|c|c|c|c|}
\hline $\mathbf{S} / \mathbf{N}$ & Items & Disagree & Undecided & Agreed \\
\hline $\mathbf{1 .}$ & I would like to use this system frequently & 13 & 2 & 35 \\
\hline $\mathbf{2}$ & I found the system unnecessarily complex. & 38 & 5 & 7 \\
\hline $\mathbf{3}$ & I thought the system was easy to use. & 15 & 0 & 35 \\
\hline $\mathbf{4}$ & $\begin{array}{c}\text { I think that I would need the support of a technical person } \\
\text { to be able to use this system. }\end{array}$ & 35 & 0 & 15 \\
\hline $\mathbf{5}$ & $\begin{array}{c}\text { I found the various functions in this system were well } \\
\text { integrated. }\end{array}$ & 13 & 2 & 35 \\
\hline $\mathbf{6}$ & I thought there was too much inconsistency in this \\
system. & 35 & 5 & 10 \\
\hline $\mathbf{7}$ & $\begin{array}{c}\text { I would imagine that most people would learn to use this } \\
\text { system very quickly }\end{array}$ & 10 & 5 & 35 \\
\hline $\mathbf{8}$ & I found the system very cumbersome to use. & 35 & 5 & 10 \\
\hline $\mathbf{9}$ & I felt very confident using the system. & 15 & 0 & 35 \\
\hline $\mathbf{1 0}$ & I needed to learn a lot of things before I could get going \\
with this system. & 35 & 10 & 5 \\
\hline
\end{tabular}




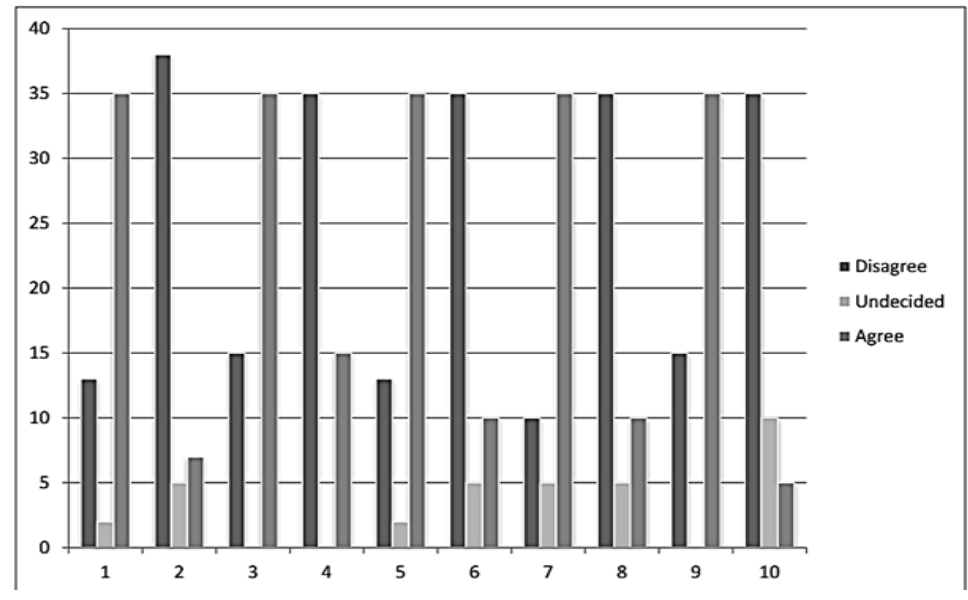

Fig.9. multiple Column Bar Chart of Usability Result

Table 2. Percentage Prevalence of infection in the body system of 10 suspected patients

\begin{tabular}{|c|c|c|}
\hline Patients & Dengue Fever (\%) & Zika Virus Infection (\%) \\
\hline Patient 1 & 29 & 71 \\
\hline Patient 2 & 26 & 74 \\
\hline Patient 3 & 80 & 20 \\
\hline Patient 4 & 56 & 44 \\
\hline Patient 5 & 74 & 26 \\
\hline Patient 6 & 20 & 80 \\
\hline Patient 7 & 45 & 55 \\
\hline Patient 8 & 32 & 68 \\
\hline Patient 9 & 45 & 55 \\
\hline $\begin{array}{c}\text { Patient } \\
\text { 10 }\end{array}$ & 60 & 40 \\
\hline
\end{tabular}

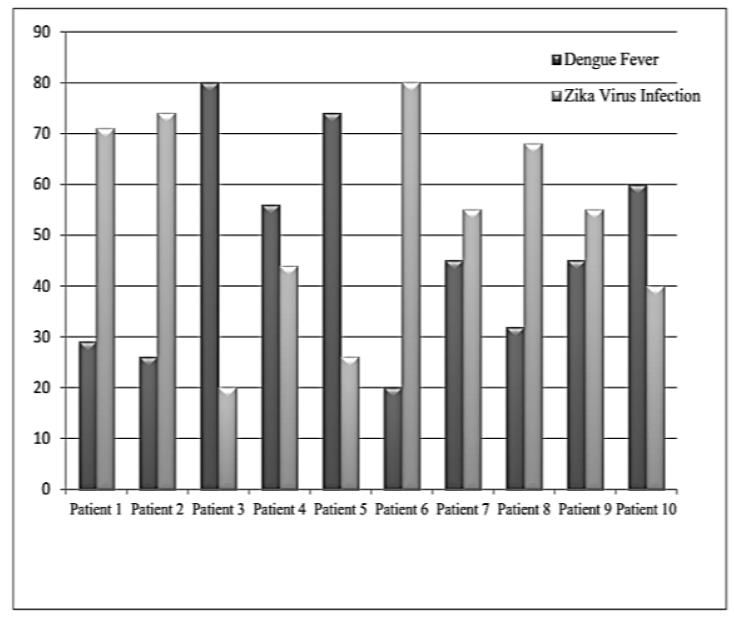

Fig.10. Infected Patients Disease Prevalence Barchart

The result shows that the system performed efficiently, effectively and was accepted by the users in the medical facilities as a friendly tool for diagnosis. An averaged response of $70.06 \%$ confirmed that NDZ system passed the usability test. $70 \%$ of respondents agreed to frequently use the system. $76 \%$ of the respondents disagreed on the system complexity indicating that the system was user friendly. $70 \%$ respondents agreed to ease of use of the prototype system. $70 \%$ of respondents disagree that technical personnel support is needed to use the system. $70 \%$ respondents agreed that the modules were well integrated. $70 \%$ respondents disagreed on the issue of system inconsistency. $70 \%$ of respondents expressed their confidence in using the system. The simulated results of differential diagnostic analysis between dengue fever and Zika Virus disease was also consistent. The process was repeated using purposive sample population of patients at the hospitals and the results were also consistent.

\section{CONCLUSION}

This paper is designed around patients who may be infected with any of the flaviruses as well as those adults who are susceptible to infections especially in the tropics where preventive healthcare is still evolving. It is an attempt to evolve an easy to use screening system for detecting the presence of any of such infection in adults. The prototype discussed is relevant in two ways. It may be used by healthcare officers to conduct a fast screening on persons. Second, it is also a "do it yourself" system which implies that any literate adult with internet connection can do a self-screening test for the viruses. The process of use is simple. In a healthcare whether or not mobile, a differential diagnostic screening test result can easily be obtained on the system often expressed as a percentage that reflects the likelihood or non-likelihood of a ZVD or a Dengue Infection in the suspected patient. $50 \%$ and above are considered significant indicators in the diagnostic process.

The diagnostic capability of the system has been tested in secondary healthcare institutions through a purposive sampling. The result obtained showed the ability of the system to fit into the requirements of the institutions for diagnosis of arbovirus diseases especially for ZVD and Dengue virus infections. The system is flexible may be expanded to accommodate more arboviral infections such as Yellow fever, Chikungunya Virus, etc. It will also address the diagnostic difficulties found in areas with arboviral disease prevalence, truncated prevalence and non-prevalence.

Academically, this paper contributes to the growing body of knowledge in the use of computerized techniques 
in finding solution to the health challenges of sudden onset such as those caused by the arboviruses.

\section{REFERENCES}

[1] National Library of Medicine. (2009). Artificial Intelligence. Retrieved August 10, 2018, from https://hsric.nlm.nih.gov/hsric_public/display_links/717

[2] Knobler, S., Mahmoud, A., Lemon, S., Mack, A., Sivitz, L., \& Oberholtzer, K. (2004). Learning from SARS: Preparing for the Next. Retrieved October 31, 2016, from https://www.ncbi.nlm.nih.gov/books/NBK92462/pdf/Boo kshelf_NBK92462.pdf

[3] Paixão, E.S., Barreto, F., Teixeira, M.G., Costa M.C., and Rodrigues L.C.(2016) "History, Epidemiology, and Clinical Manifestations of Zika: A Systematic Review.", American journal of Public Health, Vol. 106, Issue 4, pp 106-112. doi: 10.2105/AJPH.2016.303112.

[4] Nigeria Center for Disease Control (2016, October). Public Health Risk Assessment Of Zika Virus In Nigeria and Interim Recommendations. Abuja: Nigeria Center for Disease Control. Accessed June 1, 2018, from http://www.ncdc.gov.ng/themes/common/docs/protocols/ 12_1478775264.pdf

[5] Centres for Disease Control and Prevention (2016) "Country Classification Technical Guidance"[online]. Available at: https://wwwnc.cdc.gov/travel/page/zikacountry-classification [Accessed 17 February 2018]

[6] Fagbami, A. H. (1979). Zika virus infections in Nigeria: virological and seroepidemiological investigations in Oyo State. J Hyg, 84, 213 - 219.

[7] Cao-Lormeau, V. M., \& Musso, D. (2014). Emerging arboviruses in the Pacific. Lancet, 384(9954), 1571-2.

[8] Duffy M. R. et al(2009) " Zika virus outbreak on Yap Island, Federated States of Micronesia", New England Journal of Medicine, Vol. 360, Issue 24, pp 536-43. doi: 10.1056/NEJMoa0805715

[9] Faria, N. R., Azevedo, R. D. S. D. S., Kraemer, M. U. G., Souza, R., Cunha, M. S., Hill, S. C. 1., ... Vasconcelos, P. F. C. (2016). Zika virus in the Americas: Early epidemiological and genetic findings. Science Journal, 352(6283), 345 - 349. Retrieved May 2, 2017, from ttp://evolve.zoo.ox.ac.uk/Evolve/Oliver_Pybus_files/Zika VirusInTheAmericas.pdf

[10] Petersen L. R., Jamieson D. J., Powers A. M., \& Honein M. A. (2016). . Zika virus. The New England Journal of Medicine, 374(16), 1552-1563.

[11] World Health Organisation (WHO). (2016, February 26). 2016 Public Health Emergency of International Concern . Retrieved December 30, 2016, from World Health Organisation: http://www.who.int/emergencies/zikavirus/en/

[12] Dowall S. D., Graham V. A., Rayner E., Atkinson B., Hall G., Watson R. J., Bosworth A., Bonney L. C., Kitchen S, \& Hewson R. (2016). A Susceptible Mouse Model for Zika Virus Infection. PLoS Neglected Tropical Diseases, 10(5). Retrieved December 1, 2017, from https://www.ncbi.nlm.nih.gov/pmc/journals/532/

[13] Onuorah, M., Adam,u A., Obi, E. I., \& Hasheem A. M. (2016). Deterministic Mathematical Model of Zika Virus. Researchjournali's Journal of Mathematics, 3(3), 1 - 16.

[14] Blázquez,A. and Saiz,J. (2016) "Neurological manifestations of Zika virus infection", World Journal of Virology Vol. 5, No.4,pp 135-143. doi: 10.5501/wjv.v5.i4.135

[15] Tripathi, S., Balasubramaniam, V.R.M.T., Brown, J. A., Mena, I., Grant, A., Bardina, S. V., ... Garc1 'a-Sastre, A., et al. (2017). A novel Zika virus mouse model reveals strain specific differences in virus pathogenesis and host inflammatory immune responses. PLOS Pathogens, 13(3), $1-19$.

[16] Olson, J.G., Ksiazek, T.G., Suhandiman, \& Triwibowo(1981) "Zika virus, a cause of fever in Central Java, Indonesia", Trans R Soc Tropical Medicine Hygiene, Vol. 75, Issue 3: pp 389-393

[17] Hole, K.R., \& Gulhane, V.S. (2014). Rule-based expert system for the diagnosis of memory loss disease. International Journal of Innovative Science, Engineering and technology Vol 1 Issue 3

[18] Patel, M.,Patel, A., \& Virparia , P (2016). Rule based expert system for viral infection diagnosis. International journal of Advancedresearch in Computer Science abd Software Engineering

[19] Ekong, V.E., Inyang, U.G., \& Onibere E.A. (2012). Intelligent decision support system for depression diagnosis based on neuro-fuzzy CBR Hybrid. Retrieved on January 20, 2018 from https://researchgat.net/publication/235435577

[20] Abu Naser S., Al-Dahdooh R., Mushtaha, A., \& El-Naffar M. (2010). Knowledge Management in ESMDA: Expert System for Medical Diagnostic Assistance. ICGST-AIML Journal, 10(1), 31 - 40 .

[21] World Health Organization (2017) "Zika virus country classification scheme"[online]. Available at http://apps.who.int/iris/bitstream/10665/254619/1/WHOZIKV-SUR-17.1-eng.pdf?ua=

[22] Kaasbøll, J. J.(1996). Aspects of object-oriented modelling: concepts for analysis and guidelines for design. Oslo: Nonnon.

[23] Mathiasen, L., munk- Nielsen, A., Nielsen, P.A and Stage, J. (2000). Object oriented Analysis and Design. Aalborg, Denmark: Marko Publishing Aps.

\section{Authors' Profiles}

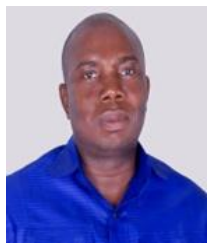

Chinecherem Umezuruike is an Assistant Lecturer in Information Technology at Kampala International University, Kampala, Uganda. His research interest is Information Systems, Intelligent Systems and Information Security

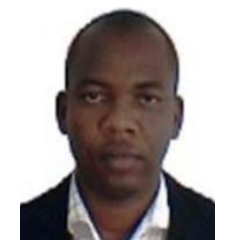

Dr Wilson Nwankwo is currently a an Associate Professor in Computer Science and Director, Professional Training and Development at Edo University Iyamho. His research interests are: Intelligent Systems; Soft computing; Cognitive systems; Analytics and Embedded systems, Wireless Networks, and high availability Database systems.

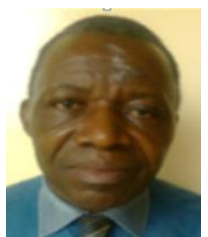

S.O. Okolie is a professor in Computer Science Department, Babcock University. His research interests are in the area of Computation and Data Structures. 


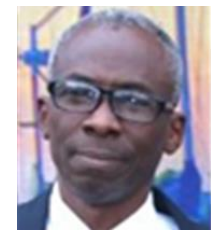

A.O. Adebayo is an associate professor of Computer Science at Babcock University. His research interests are Information Security and Information Systems. He has published scientific articles in several journals of international repute.

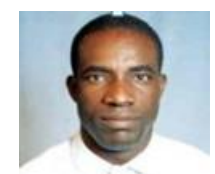

J.V. Joshua is an associate professor of Computer Science at Babcock University. His research interests are Software Engineering and Information Systems. He has published scientific articles in several journals of international repute.

H.N. Ngugi is an Assistant Lecturer in Computer Science at Kampala International University, Kampala Uganda. Her research interests are: Soft computing and Information Systems.

How to cite this paper: Chinecherem Umezuruike, Wilson Nwankwo, Samuel O. Okolie, Adewale O. Adebayo, Joshua V. Jonah, Habiba Ngugi, "Health Informatics System for Screening Arboviral Infections in Adults", International Journal of Information Technology and Computer Science(IJITCS), Vol.11, No.3, pp.10-22, 2019. DOI: 10.5815/ijitcs.2019.03.02 